\section{$\Rightarrow$ GLOMERULONEPHRITIS}

\section{Not your usual parietal cell}

In the glomerulus, parietal epithelial cells (PECs) are well known for their contribution, along with podocytes, to sclerotic lesions in primary focal and segmental glomerulonephritis (FSGS).

Now, Kuppe, Moeller and colleagues report that parietal cells with features of proximal tubular cells (PTCs) might "represent a hotspot for lesion formation in the early stages of glomerular disease."

In response to injury, PTCs can transiently activate a scattered tubular cell (STC) transcriptional programme that enhances cellular

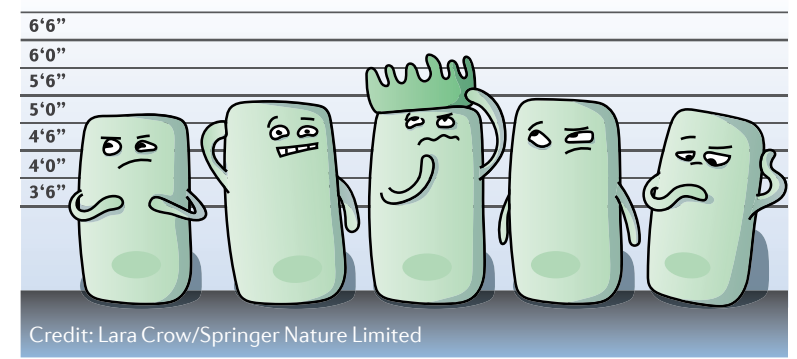

activation and proliferation; this response is thought to contribute to tubular cell regeneration. In human kidney biopsy samples, in addition to the classical flat PECs that line the Bowman's capsule, the researchers identified a group of PECs at the PEC-PTC border that co-expressed keratin 7 and 19, two STC phenotype markers - these cells were termed intermediate PECs (iPECs). In some glomeruli, PTC-like cells, termed cuboidal PECs, extended from the proximal tubule into the Bowman's capsule. In mice, expression of the STC marker cyclin D1 identified iPECs, which were located between flat and cuboidal PECs near the proximal tubule.

The researchers used transgenic mice to fluorescently label either all PECs, or only cuboidal PECs and iPECs, for lineage tracing studies. "In ageing mice, we found no evidence that flat PECs regenerate cuboidal PECs or vice versa, which argues against previous reports of a potential progenitor niche at this location," remarks Moeller.

Following FSGS induction in Thyl.1 mice, cuboidal PECs and
iPECs expressed the highest levels of proliferation and activation markers in the glomerulus. Moreover, in the 5/6 nephrectomy and DOCA/salt model of FSGS, both sclerotic and proliferative lesions contained PTC-like cells (that is, cuboidal PECs and iPECs). Finally, in human renal biopsy samples of early FSGS recurrences, cells that expressed keratin 7 , a marker found in human iPECs, were particularly abundant in tip lesions, in which adhesions are formed near the tubular pole.

"Our findings in mice and humans demonstrate that tip lesions originate from iPECs," concludes Kuppe.

"We plan to investigate what promotes the migration of proximal tubule cells into the Bowman's capsule and whether the presence of these cells is a risk factor for renal lesion formation," explains Moeller.

Monica Wang

ORIGINAL ARTICLE Kuppe, C. et al. Novel parietal epithelial cell subpopulations contribute to focal segmental glomerulosclerosis and glomerular tip lesions. Kidney Int. https://doi.org/ 10.1016/j.kint.2019.01.037 (2019)

\title{
Macrophages support kidney and vasculature assembly in development
}

Available evidence suggests that macrophages can promote the growth and maintenance of renal tissue; however, the exact origin and roles of macrophages in the developing kidney are unclear. New findings suggest that macrophages have dual roles in defining the metanephric field and in promoting vascular connectivity in the developing kidney. "Our findings indicate that macrophages prune the rostral domain of the early nephron progenitor population," says David Munro. "In addition, deletion of macrophages resulted in fewer connections being formed between blood vessels, suggesting that macrophages are involved in shaping the network of blood vessels in the developing kidney."

Using imaging to characterize macrophage distribution, the researchers show that macrophages localize near the metanephric mesenchyme as it condenses at the caudal aspect of the Wolffian duct. However, these macrophages largely avoid the caudal metanephric mesenchyme and instead mainly localize alongside rostral clusters of SIX2+ nephron progenitor cells, where the researchers propose they might clear rostral SIX $2^{+}$cells. In support of this hypothesis, deletion of macrophages led to persistence of rostral nephrogenic progenitors and delayed ureteric bud development.

The researchers further showed that the developing kidney contains both an early population of macrophages and a later-arriving population of myeloid cells. Imaging studies showed macrophages interacting with the developing renal vasculature. "A surprisingly high percentage of macrophages were wrapped around newly forming blood vessels,"

deletion of
macrophages
resulted
in fewer
connections
being formed
between blood
vessels

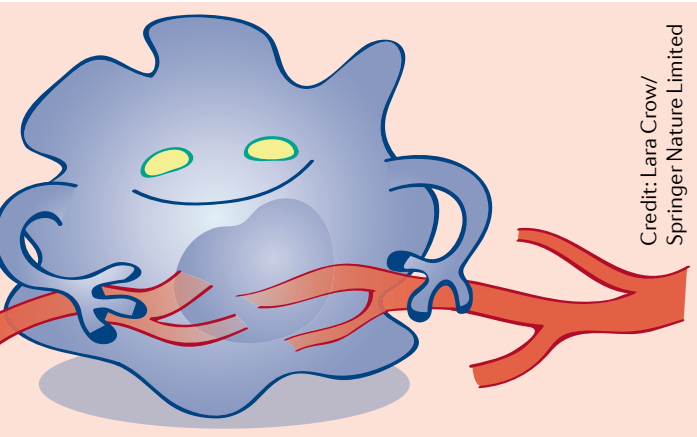

explains Munro. Single-cell RNA sequencing showed that in addition to being enriched for mRNAs linked to phagocytosis, kidney macrophages expressed mRNAs linked to vasculature development. Finally, the researchers showed that depleting macrophages induced higher numbers of unconnected endothelial structures, demonstrating a role for the macrophages in the production or maintenance of endothelial connections in the developing kidney.

Susan J. Allison

ORIGINAL ARTICLE Munro, D. A. D. et al.

Macrophages restrict the nephrogenic field and promote endothelial connections during kidney development. eLife 8, e43271 (2019) 